\title{
GRAVITY SOLITARY WAVES BY MINIMIZATION: AN UNCOUNTABLE FAMILY
}

\author{
BORIS BUFFONI
}

\begin{abstract}
We improve and simplify the minimization method for solitary waves in two cases: firstly, when the surface tension is weak (that is, the Bond number is $<1 / 3$ ) and the depth is finite, and secondly, when the depth is infinite. In a previous work on the first case, minimizers were shown to exist for a sequence tending to 0 of values of the horizontal impulse. The main difficulty is that strict subadditivity in the concentration-compactness method is unsettled. Here we observe in both examples that strict subadditivity nevertheless holds for a set of horizontal impulses of positive measure and the related propagation speeds are estimated from above.
\end{abstract}

\section{Introduction}

In the Euclidean plane, consider an horizontal layer of perfect fluid that is inviscid, of constant density and submitted to constant downward gravity. The upper boundary is free, submitted to surface tension and described by the graph of a map $(\tau, x) \rightarrow \eta(\tau, x)$, where $\tau$ is time and $x$ the horizontal variable. Its depth is either finite with flat horizontal bottom (first example) or infinite (second example). We are interested in the case $\eta \rightarrow 0$ as $x \rightarrow \pm \infty$ and the flow is irrotational.

2000 Mathematics Subject Classification. 76B15, 76B25, 76E17, 35J20.

Key words and phrases. Capillary-gravity water waves, solitary waves, stability, variational methods.

Supported by a grant of the Swiss National Science Foundation. 
As observed by Zakharov [17], the problem has an Hamiltonian structure: if $(\tau, x) \rightarrow \xi(\tau, x)$ is the trace of the velocity potential on the upper surface, then, formally,

$$
\partial_{\tau} \eta=\partial_{\xi} \mathcal{H}(\eta, \xi), \quad \partial_{\tau} \xi=-\partial_{\eta} \mathcal{H}(\eta, \xi),
$$

where $(\eta, \xi) \rightarrow \mathcal{H}(\eta, \xi)$ is the nonlinear and nonlocal functional that gives the total energy (depending on the velocity, gravity and surface tension), and $\partial_{\xi}$ and $\partial_{\eta}$ denote functional derivatives.

The total horizontal momentum $\mathcal{P}(\eta, \xi)$ (that is, the integral of the horizontal velocity below the surface) is preserved, which means that $\partial_{\tau} \mathcal{P}(\eta, \xi)=0$. The profiles $\left(\eta_{0}, \xi_{0}\right):=(\eta(0, \cdot), \xi(0, \cdot))$ at times $\tau=0$ of solitary waves with given $\mathcal{P}$ can be found as critical points of $\left(\eta_{0}, \xi_{0}\right) \rightarrow \mathcal{H}\left(\eta_{0}, \xi_{0}\right)$ under the constraint $\mathcal{P}\left(\eta_{0}, \xi_{0}\right)=\mathcal{P}_{0}$, where $\mathcal{P}_{0}$ is any small positive constant. Given $\eta_{0}$, let $\xi_{0}=\xi_{0}\left(\eta_{0}\right)$ minimize $\xi_{0} \rightarrow H\left(\eta_{0}, \xi_{0}\right)$ under the constraint $\mathcal{P}\left(\eta_{0}, \xi_{0}\right)=\mathcal{P}_{0}$. Solitary waves can then be found by minimizing the functional $\eta_{0} \rightarrow \mathcal{H}\left(\eta_{0}, \xi_{0}\left(\eta_{0}\right)\right)$. After a nonlocal transformation $\eta_{0} \rightarrow u$ [1], [2], [10] and considering some appropriate multiple $\mu>0$ of $\mathcal{P}_{0}$, we are lead to the following nonlinear functional

$$
u \rightarrow \mathcal{J}_{\mu}(u):=\mathcal{K}(u)+\frac{\mu^{2}}{\mathcal{L}(u)}
$$

defined for $u \neq 0$ in a small ball

$$
U=\left\{u \in W^{2,2}(\mathbb{R}):\|u\|_{W^{2,2}(\mathbb{R})}<r\right\}, \quad r>0 .
$$

We assume $\mathcal{K}$ and $\mathcal{L}$ to be $C^{1}$ on $U$ endowed with the $W^{2,2}(\mathbb{R})$-topology (this can be proved for the two particular examples). The critical points of $\mathcal{J}_{\mu}$ satisfy

$$
\mathcal{K}^{\prime}(u)=\frac{\mu^{2}}{\mathcal{L}(u)^{2}} \mathcal{L}^{\prime}(u)
$$

The stability problem for $\mathcal{J}_{\mu}$ is to show that its set of minimizers is not empty and that each minimizing sequence tends to the set of minimizers. See [4] for more explanation and the relationship between $\mathcal{J}_{\mu}$ and Smale's amended potentials [15], [16]. In what follows, the spatial variable $t$ is related to $x$ by some nonlocal transformation.

In the first example, $\mathcal{K}(u)$ and $\mathcal{L}(u)$ are given by

$$
\mathcal{K}(u)=\int_{\mathbb{R}}\left\{\beta \sqrt{{u^{\prime}}^{2}+(1+N u)^{2}}-\beta(1+N u)+\frac{1}{2} u^{2}(1+N u)\right\} d t
$$

and

$$
\mathcal{L}(u)=\frac{1}{2} \Lambda \int_{\mathbb{R}} u \cdot N u d t,
$$

where $\beta \in(0,1 / 3)$ is a fixed parameter, $N: W^{1,2}(\mathbb{R}) \rightarrow L^{2}(\mathbb{R})$ is the non-local operator defined via Fourier's transform by

$$
\widehat{N u}(s)=\frac{s \cosh s}{\sinh s} \widehat{u}(s),
$$


and the constant

$$
\Lambda=\min \left\{\left(\beta s^{2}+1\right) \frac{\sinh s}{s \cosh s}: s \geq 0\right\}>0
$$

enforces the normalization property (2.1) below (we $\operatorname{set} \sinh (s) / s=1$ at $s=0$ ).

The critical points of $\mathcal{J}_{\mu}$ correspond in this example to solitary capillarygravity water waves on a two-dimensional ocean of finite depth when the surface tension is weak. The parameter $\beta \in(0,1 / 3)$ is the Bond number (a dimensionless combination of various physical quantities, including gravity and surface tension, that is fixed in all what follows). The parameter $\mu>0$ is proportional to the total horizontal impulse and the propagation speed is proportional to $\mu / \mathcal{L}(u)$ (the proportionality constant being independent of $\mu$ and the critical point $u$ ).

For this example, it can be shown like in [4] that the stability problem is indeed related to conditional stability of solitary water waves. See also [14] for an approach to stability based on center-manifold theory and that introduced the main concepts used in [4]-[6].

In the second example,

$$
\begin{aligned}
\mathcal{K}(u) & =\int_{\mathbb{R}} \sqrt{{u^{\prime}}^{2}+\left(1+\mathcal{H} u^{\prime}\right)^{2}}-\left(1+\mathcal{H} u^{\prime}\right) d t+\frac{1}{2} \int_{\mathbb{R}} u^{2}\left(1+\mathcal{H} u^{\prime}\right) d t \\
& =\int_{\mathbb{R}} \frac{u^{\prime 2} d t}{\sqrt{u^{\prime 2}+\left(1+\mathcal{H} u^{\prime}\right)^{2}}+\left(1+\mathcal{H} u^{\prime}\right)}+\frac{1}{2} \int_{\mathbb{R}} u^{2}\left(1+\mathcal{H} u^{\prime}\right) d t, \\
\mathcal{L}(u) & =\int_{\mathbb{R}} u \mathcal{H} u^{\prime} d t,
\end{aligned}
$$

where $\mathcal{H}: L^{2}(\mathbb{R}) \rightarrow L^{2}(\mathbb{R})$ is the Hilbert transform, defined by

$$
\widehat{\mathcal{H} v}(s)=-i \operatorname{sgn}(s) \widehat{v}(s), \quad \widehat{v}(s)=\frac{1}{\sqrt{2 \pi}} \int_{\mathbb{R}} e^{-i t s} v(t) d t .
$$

If $r$ is small enough, the denominator in $\mathcal{K}$ is positive, thanks to $\|u\|_{L^{\infty}(\mathbb{R})} \leq$ Const $\|u\|_{W^{1,2}(\mathbb{R})}$ by a standard Sobolev's embedding, and to the fact that the Hilbert transform is a bounded operator in $L^{2}(\mathbb{R})$ (in fact $\mathcal{H}$ preserves the norm).

Concerning the second example, Iooss and Kirrmann [11] proved the existence of at least two even solitary waves on the surface of a two-dimensional ocean of infinite depth, with the help of normal form theory for reversible infinitedimensional dynamical systems. Later, in [5], we showed the existence by a variational method of a solitary wave, offering in this way an alternative method. However, the variational nature of the solution was unclear because, in the proof, there was a loss of information when taking limits of minimizing sequences.

In this paper we follow the minimization approach of [4], [6], so that the solitary waves we now find are really minimizers of the functional $\mathcal{J}_{\mu}$. The present approach is also more efficient than the one in [6] because it leads to a minimizer of $\mathcal{J}_{\mu}$ for a set of values of $\mu$ that is of positive Lebesgue's measure, 
whereas in [6] the set was only shown to be countable. Moreover, for these values of $\mu$, the stability problem can be solved. The main ingredient is the concentration-compactness method [12], [13], as in the stability analysis in [9].

Let us now state precisely the stability property (ST) that we shall prove in both examples for a measurable set of values of $\mu$ that has positive Lebesgue's measure (assuming moreover that $r$ is small enough).

Stability property (ST) at $\mu>0$. The set of minimizers of $\mathcal{J}_{\mu}$ defined on $U \backslash\{0\}$ is not empty and, for all minimizing sequences $\left\{u_{n}\right\}$ satisfying

$$
\limsup _{n \rightarrow \infty}\left\|u_{n}\right\|_{W^{2,2}(\mathbb{R})}<r
$$

the $L^{2}(\mathbb{R})$-distance between $u_{n}$ and the set of minimizers tends to 0 as $n \rightarrow \infty$.

It is explained in [4] that, in the first example, property (ST) indeed implies some kind of weak conditional stability of the set of solitary waves corresponding to the minimizers of $\mathcal{J}_{\mu}$.

In each example, once it is known that $\mathcal{J}_{\mu}$ has a minimizer $u \in U \backslash\{0\}$ (with $r$ small), more information on $u$ could be obtained by studying the equation $\mathcal{K}^{\prime}(u)=\left(\mu^{2} / \mathcal{L}(u)\right) \mathcal{L}^{\prime}(u)$, like the regularity of $u$ and its asymptotic behavior (see e.g. [3]). However, it is unsettled if $t \rightarrow u(t)$ is even with respect to some $t$, like the solitary waves obtained in [11] in the second example.

Note added in proof. In a joint work with Mark Groves and Shu-Ming Sun (B. Buffoni, M. Groves and S.-M. Sun, Existence and conditional energetic stability of three-dimensional fully localised solitary gravity-capillary water waves, preprint), we establish the existence and conditional energetic stability of 3D fully-localized waves for all small values of the horizontal impulse, extending in this way a previous work by M. Groves and S.-M. Sun (M. Groves and S.-M. Sun, Fully localised solitary-wave solutions of the three-dimensional gravity-capillary water-wave problem, Arch. Rational Mech. Anal. 188 (2008), 1-91). In this case, we carefully analyze the minimizing sequences of some penalized functional and build a particular minimizing sequence made of functions behaving appropriately at infinity. This allows us to show strict subadditivity.

\section{List of hypotheses}

Remember that $\mathcal{K}$ and $\mathcal{L}$ are assumed to be $C^{1}$ on $U$ endowed with the $W^{2,2}(\mathbb{R})$-topology. The following additional hypotheses are satisfied in both examples.

Regularity hypothesis $(\mathbf{R})$ on $\mathcal{K}, \mathcal{L} . \mathcal{K}$ and $\mathcal{L}$ are continuous on $U$ endowed with the topology induced from $L^{2}(\mathbb{R})$. 
REMARK 2.1. This hypothesis holds true for both examples (if $r$ is small enough) because of the estimate

$$
\left\|u^{\prime}\right\|_{L^{\infty}(\mathbb{R})}+\|N u\|_{L^{\infty}(\mathbb{R})}+\left\|\mathcal{H} u^{\prime}\right\|_{L^{\infty}(\mathbb{R})} \leq \text { Const }\|u\|_{W^{2,7 / 4}(\mathbb{R})}
$$

and the fact that convergence to 0 in $L^{2}(\mathbb{R})$ of any sequence bounded in $W^{2,2}(\mathbb{R})$ implies convergence to 0 in $W^{2,7 / 4}(\mathbb{R})$. In fact, in both examples, $\mathcal{L}$ is quadratic and $\mathcal{K}$ is analytical if $r$ is small enough, but only continuity is used in this paper. As $U$ is bounded in $W^{2,2}(\mathbb{R})$, hypothesis $(\mathrm{R})$ implies that $\mathcal{K}$ and $\mathcal{L}$ are continuous on $U$ endowed with the topology induced from $W^{2, s}(\mathbb{R})$ for any fixed $0<s<2$.

Coercivity hypothesis (C) of $\mathcal{K}, \mathcal{L}$. For all $u \in U \backslash\{0\}$

$$
0<\mathcal{L}(u) \leq \text { Const } \quad \text { and } \quad \mathcal{K}(u) \geq \text { Const } \int_{\mathbb{R}} u^{2} d t
$$

for some positive constant.

REMARK 2.2. It clearly holds in both examples if $r$ is small enough.

Hypothesis (BMS): existence of a sufficiently Bounded Minimizing Sequence. There exists a minimizing sequence $\left\{u_{n}\right\} \subset U \backslash\{0\}$ of $\mathcal{J}_{\mu}$ such that

$$
\limsup _{n \rightarrow \infty}\left\|u_{n}\right\|_{W^{2,2}(\mathbb{R})}<r .
$$

REMARK 2.3. If $r$ is small enough, such a minimizing sequence exists in both examples for all values of $\mu$ that are small enough. However, our proof that such a minimizing sequence exists is involved. For the first example (where $\beta \in(0,1 / 3))$, the proof is almost the same as the proof of Lemma 16 in [4] for the case $\beta \geq 1 / 3$. The proof for the second example is similar, but slightly more complicated (see the Appendix). Note that, in both case, the proof relies heavily on Theorem 1 in [4], which is related to the previous works [7], [8]. We shall need the existence of such a minimizing sequence to show that the set of minimizers is not empty for many values of $\mu$.

Hypothesis (NV): non vanishing of every minimizing sequence $\left\{u_{n}\right\}$ $\subset U \backslash\{0\}$ of $\mathcal{J}_{\mu}$.

$$
\liminf _{n \rightarrow \infty}\left\{\max _{t}\left|u_{n}(t)\right|+\max _{t}\left|u_{n}^{\prime}(t)\right|\right\}>0 .
$$

REMARK 2.4. For such a sequence $\left\{u_{n}\right\}$, (3.1) below holds too if each $u_{n}$ is shifted in $t$ appropriately. An important step in the concentration-compactness method is to prove it for every minimizing sequence (see [12], [13] where the idea is explained in a more general setting). The proof for the first example can be found in [4] and the proof for the second example is similar ( $\mu$ and $r$ small enough).

$$
\text { Let } c(\mu)=\inf \left\{\mathcal{J}_{\mu}(w): w \in U \backslash\{0\}\right\}
$$




\section{Subcriticallity hypothesis (SUB-C) on $c$.}

$$
\lim _{\mu \rightarrow 0^{+}} \frac{c(\mu)}{\mu}=2 \text { and } \exists \mu_{0}>0 \exists \kappa>0 \forall \mu \in\left(0, \mu_{0}\right] c(\mu) \leq 2 \mu-\kappa \mu^{3} .
$$

Remarks 2.5. This implies $\mathcal{L}(0)=0$. The value 2 appearing as limit in (SUB-C) is, in both examples, related to the fact that

$$
\inf _{u \neq 0} \frac{\mathcal{K}^{\prime \prime}(0)(u, u)}{2 \mathcal{L}(u)}=1 .
$$

Indeed, in the first example, $\mathcal{K}^{\prime \prime}(0)(u, u)=\int_{\mathbb{R}}\left(\beta u^{\prime 2}+u^{2}\right) d t$ and in the second $\mathcal{K}^{\prime \prime}(0)(u, u)=\int_{\mathbb{R}}\left(u^{\prime 2}+u^{2}\right) d t$. In the first example, $\Lambda$ in the definition of $\mathcal{L}$ is chosen in such a way that (2.1) holds. Property (2.1) also holds in the second example, as it is easy to check by working with Fourier's transform. (SUB-C) holds for the first example, as proved in [6] with the help of a "test function". In the Appendix, we shall sketch the proof that (SUB-C) holds in the second example too. In both examples, (SUB-C) is in fact used to prove that hypothesis (BMS) holds and to show the non-vanishing hypothesis (NV). Finally, in the proof of our main result, it is used to check that strict subadditivity holds for many values of $\mu$ (see (SUB-A) below).

Splitting hypothesis (SP). If the sequence $\left\{u_{n}\right\} \subset U$ satisfies (1.1) and converges weakly to some $w_{\infty} \in U$, then, replacing $\left\{u_{n}\right\}$ by one of its subsequence if necessary, there exist two sequences $\left\{u_{1, n}\right\} \subset U$ and $\left\{u_{2, n}\right\} \subset U$ such that:

(a) for all $n \in \mathbb{N}$ and $j \in\{1,2\}$, the function $u_{j, n}$ has compact support $\operatorname{supp}\left(u_{j, n}\right) \subset \mathbb{R}$

(b) $\lim _{n \rightarrow \infty} \operatorname{dist}\left(\operatorname{supp}\left(u_{1, n}\right), \operatorname{supp}\left(u_{2, n}\right)\right)=\infty$;

(c) $\lim _{n \rightarrow \infty}\left(\mathcal{K}\left(u_{n}\right)-\mathcal{K}\left(u_{1, n}\right)-\mathcal{K}\left(u_{2, n}\right)\right)=0$;

(d) $\lim _{n \rightarrow \infty}\left(\mathcal{L}\left(u_{n}\right)-\mathcal{L}\left(u_{1, n}\right)-\mathcal{L}\left(u_{2, n}\right)\right)=0$;

(e) $\lim _{n \rightarrow \infty}\left\|u_{1, n}+u_{2, n}-u_{n}\right\|_{L^{2}(\mathbb{R})}=0$;

(f) $u_{n} \rightarrow w_{\infty}$ weakly in $W^{2,2}(\mathbb{R})$ and $\lim _{n \rightarrow \infty}\left\|u_{1, n}-w_{\infty}\right\|_{L^{2}(\mathbb{R})}=0$ for some $w_{\infty} \in W^{2,2}(\mathbb{R})$.

REMARKS 2.6. The splitting property is a key ingredient in the concentration-compactness method [12], [13]. It holds for the first example if $r>0$ is small enough, as proved in Theorem 4 in [4]. It also holds for the second example, the proof being the same, but equation (19) in [5] is used instead of equation (15) in [4]. In $3 U$, all the norms $W^{s, 2}(\mathbb{R})$ are equivalent for $0 \leq s<2$. Hence, in the statement, the $L^{2}(\mathbb{R})$-norm can be replaced by any $W^{s, 2}(\mathbb{R})$-norm with $0<s<2$. When applying the splitting property, the point is usually to show that $\left\{u_{2, n}\right\}$ converges to 0 in $L^{2}(\mathbb{R})$. 


\section{Results}

The following property is also a key ingredient of the concentration-compactness method. It appears as hypothesis in the lemma below and will be shown to hold in both examples for a measurable set of values of $\mu$ that has positive Lebesgue's measure (see the proof of the theorem below).

Property (SUB-A) at $\mu>0$ : strict subadditivity. $c(\mu)<c\left(\mu_{1}\right)+c\left(\mu_{2}\right)$ for all $\mu_{1}, \mu_{2}>0$ such that $\mu_{1}+\mu_{2}=\mu$.

REMARK 3.1. It is unsettled in our examples if it holds for all small $\mu$.

The concentration-compactness lemma can be stated as follows (see [4], which deals with $\mathcal{J}_{\mu}$, and [12], [13] for the general case):

Lemma 3.2. Let $\mathcal{K}, \mathcal{L}$ satisfy the hypotheses $(\mathrm{R}),(\mathrm{C})$ and $(\mathrm{SP})$ above, $\mu>$ 0 satisfy the strict subadditivity property (SUB-A) and $\left\{u_{n}\right\}$ be a minimizing sequence of $\mathcal{J}_{\mu}$ that satisfies (1.1) and

$$
\liminf _{n \rightarrow \infty}\left\{\left|u_{n}(0)\right|+\left|u_{n}^{\prime}(0)\right|\right\}>0 .
$$

Then $\left\{u_{n}\right\}$ converges in $L^{2}(\mathbb{R})$, up to a subsequence.

ProOF. Let $\left\{u_{n}\right\}$ converge weakly to $w_{\infty}$ (after extracting a subsequence if necessary). Consider the two sequences given by the splitting property. Observe that

$$
\lim _{n \rightarrow \infty} \mathcal{K}\left(u_{1, n}\right)=\mathcal{K}\left(w_{\infty}\right) \text { and } \lim _{n \rightarrow \infty} \mathcal{L}\left(u_{1, n}\right)=\mathcal{L}\left(w_{\infty}\right)
$$

by (f) of the splitting property and by the regularity hypothesis $(\mathrm{R})$ on $\mathcal{K}, \mathcal{L}$. Moreover, taking subsequences if necessary, the sequences $\left\{\mathcal{K}\left(u_{n}\right)\right\}$ and $\left\{\mathcal{L}\left(u_{n}\right)\right\}$ can be assumed to converge (since $\left\{u_{n}\right\}$ is a minimizing sequence, $\mathcal{K}$ remains bounded along it).

First consider the case

$$
L:=\lim _{n \rightarrow \infty} \mathcal{L}\left(u_{n}\right)>\mathcal{L}\left(w_{\infty}\right) .
$$

Set

$$
\mu_{1}=\mu \frac{\lim _{n \rightarrow \infty} \mathcal{L}\left(u_{1, n}\right)}{L}=\mu \frac{\mathcal{L}\left(w_{\infty}\right)}{L} \quad \text { and } \quad \mu_{2}=\mu \frac{\lim _{n \rightarrow \infty} \mathcal{L}\left(u_{2, n}\right)}{L} .
$$

Since $w_{\infty} \not \equiv 0$, we know that $\mu_{1}, \mu_{2}>0$ with $\mu_{1}+\mu_{2}=\mu$ (see hypothesis (C) and (3.1)). By strict subadditivity, we get the contradiction

$$
\begin{aligned}
c(\mu) & <c\left(\mu_{1}\right)+c\left(\mu_{2}\right) \\
& \leq \lim _{n \rightarrow \infty}\left\{\mathcal{K}\left(u_{1, n}\right)+\mu_{1}^{2} \mathcal{L}\left(u_{1, n}\right)^{-1}\right\}+\lim _{n \rightarrow \infty}\left\{\mathcal{K}\left(u_{2, n}\right)+\mu_{2}^{2} \mathcal{L}\left(u_{2, n}\right)^{-1}\right\} \\
& =\lim _{n \rightarrow \infty} \mathcal{K}\left(u_{1, n}\right)+L^{-2} \mu^{2} \lim _{n \rightarrow \infty} \mathcal{L}\left(u_{1, n}\right)+\lim _{n \rightarrow \infty} \mathcal{K}\left(u_{2, n}\right)+L^{-2} \mu^{2} \lim _{n \rightarrow \infty} \mathcal{L}\left(u_{2, n}\right) \\
& =\lim _{n \rightarrow \infty}\left\{\mathcal{K}\left(u_{n}\right)+\mu^{2} \mathcal{L}\left(u_{n}\right)^{-1}\right\}=c(\mu) .
\end{aligned}
$$


Hence we have proved that $L=\mathcal{L}\left(w_{\infty}\right)$. From $\mathcal{K} \geq 0$ on $U$, we deduce that

$$
\begin{aligned}
c(\mu) & \leq \mathcal{K}\left(w_{\infty}\right)+\mu^{2} \mathcal{L}\left(w_{\infty}\right)^{-1}=\lim _{n \rightarrow \infty}\left\{\mathcal{K}\left(u_{1, n}\right)+\mu^{2} \mathcal{L}\left(u_{n}\right)^{-1}\right\} \\
& \leq \lim _{n \rightarrow \infty}\left\{\mathcal{K}\left(u_{1, n}\right)+\mathcal{K}\left(u_{2, n}\right)+\mu^{2} \mathcal{L}\left(u_{n}\right)^{-1}\right\} \\
& =\lim _{n \rightarrow \infty}\left\{\mathcal{K}\left(u_{n}\right)+\mu^{2} \mathcal{L}\left(u_{n}\right)^{-1}\right\}=c(\mu),
\end{aligned}
$$

which shows that the minimum of $\mathcal{J}_{\mu}$ in $U$ is attained at $w_{\infty}$. Since, by the coercivity hypothesis $(\mathrm{C}), \mathcal{K}\left(u_{2, n}\right) \geq$ Const $\int_{\mathbb{R}} u_{2, n}^{2} d t$ (for some positive constant), we also get that $\left\|u_{2, n}\right\|_{L^{2}(\mathbb{R})} \rightarrow 0$ and $\left\|u_{n}-w_{\infty}\right\|_{L^{2}(\mathbb{R})} \rightarrow 0$.

Our main result is

TheOREM 3.3. Let $\mathcal{K}, \mathcal{L}$ satisfy the hypotheses $(\mathrm{R}),(\mathrm{C})$ and $(\mathrm{SP})$, and $c$ satisfy the subcriticallity hypothesis (SUB-C). Suppose further that, for all small enough $\mu$, hypotheses (BMS) and (NV) hold. Then there exists a Borelian set $\mathcal{S} \subset(0, \infty)$ such that

(a) $\mathcal{S} \cap(0, \delta]$ has positive Lebesgue's measure for all $\delta>0$;

(b) for all $\mu \in \mathcal{S}$ and all minimizing sequences $\left\{u_{n}\right\}$ of $\mathcal{J}_{\mu}$ in $U \backslash\{0\}$ satisfying (1.1) and (3.1), there exists a subsequence that converges in $U \backslash\{0\}$ with respect to the $L^{2}(\mathbb{R})$-distance;

(c) the stability property (ST) above holds for all $\mu \in \mathcal{S}$;

(d) $\mu / \mathcal{L}(w)<1$ for all $\mu \in \mathcal{S}$ and all $w \in U \backslash\{0\}$ such that $\mathcal{J}_{\mu}(w)=c(\mu)$, where $c(\mu)$ is the infimum of $\mathcal{J}_{\mu}$ on $U \backslash\{0\}$ (remember that $\mu / \mathcal{L}(w)$ is proportional to the speed of propagation).

By interpolation, the convergence of the subsequence in (b) also holds with respect to the $W^{1,2}(\mathbb{R})$ and the $W^{1, \infty}(\mathbb{R})$ distances. Its limit cannot be the function 0 , because of (3.1), and it is therefore a minimizer of $\mathcal{J}_{\mu}$ (thanks to the regularity hypothesis $(\mathrm{R}))$.

In [6], a weaker version of the previous theorem was proved for finite depth and weak surface tension; namely an analogous set to $\mathcal{S}$ was obtained that satisfies the third conclusion of the Theorem but was only shown to contain a sequence converging to 0 , without estimate on $\mu / \mathcal{L}(w)$.

Proof. In the concentration-compactness method [12], [13], an important intermediate result is strict subadditivity for all small $\mu$. It is unknown for the examples considered in this paper if it does indeed hold for all small $\mu$. The present improvement relies on the observation that if, for some small $\mu>0$, the function $s \rightarrow c(s) / s$ reaches its strict minimum on $(0, \mu]$ exactly at $\mu$, then $c(\mu)<c\left(\mu_{1}\right)+c\left(\mu_{2}\right)$ for all $\mu_{1}, \mu_{2}>0$ with $\mu_{1}+\mu_{2}=\mu$. Indeed

$$
c(\mu)=\mu_{1} \frac{c(\mu)}{\mu}+\mu_{2} \frac{c(\mu)}{\mu}<\mu_{1} \frac{c\left(\mu_{1}\right)}{\mu_{1}}+\mu_{2} \frac{c\left(\mu_{2}\right)}{\mu_{2}}=c\left(\mu_{1}\right)+c\left(\mu_{2}\right) .
$$


Let $\mathcal{S} \subset\left(0, \mu_{0}\right]$ be the set of such values of $\mu\left(\mu_{0}>0\right.$ small enough).

Let us first prove (b) and (c). By Lemma 3.2, if $\mu \in \mathcal{S}$ and $\left\{u_{n}\right\}$ is any minimizing sequence of $\mathcal{J}_{\mu}$ in $U \backslash\{0\}$ satisfying (1.1) and (3.1), then $\left\{u_{n}\right\}$ converges (up to a subsequence) in $U \backslash\{0\}$ with respect to the $L^{2}(\mathbb{R})$-distance. This proves (b).

If $\left\{u_{n}\right\}$ satisfies

$$
\liminf _{n \rightarrow \infty}\left\{\max _{t}\left|u_{n}(t)\right|+\max _{t}\left|u_{n}^{\prime}(t)\right|\right\}>0
$$

instead of (3.1), then the conclusion holds after shifting in $t$ each $u_{n}$. This shows stability for $\mu \in \mathcal{S}$ and proves (c). See [4], [6].

The subcriticallity hypothesis (SUB-C) shows that $\lim _{s \rightarrow 0^{+}} c(s) / s=2^{-}$and that $I=\left\{c(s) / s: 0<s \leq \mu_{0}\right\}$ is a non-trivial interval (thanks to the continuity of $c$, see below) that is included in $(0,2)$. For $t \in I$, define $h(t)=\min \{\tau \in$ $\left.\left(0, \mu_{0}\right]: c(\tau) / \tau=t\right\}$, so that $\mathcal{S}=\{h(t): t \in I\}$. The map $t \rightarrow h(t)$ is a bijection from $I$ to $\mathcal{S}$, its inverse being the map $s \rightarrow c(s) / s$ restricted to $\mathcal{S}$. This shows that $\mathcal{S}$ is uncountable and, moreover, that $0 \in \overline{\mathcal{S}}$ because $h(t) \rightarrow 0^{+}$as $t \rightarrow 2^{-}$.

This remark can be improved as follows. First observe that the function $s \rightarrow c(s) / s$ is locally Lipschitz on $\left(0, \mu_{0}\right]$. Indeed, for all $\delta \in\left(0, \mu_{0}\right)$, the map $c$ is Lipschitz on $\left[\delta, \mu_{0}\right]$ as the infimum of the family of equi-Lipschitz functions $s \rightarrow \mathcal{J}_{s}(w)$ (here $w$ such that $\mathcal{L}(w)>\delta / 4$ is seen as a parameter for the family):

$$
c(s)=\inf \left\{\mathcal{J}_{s}(w):\|w\|_{W^{2,2}(\mathbb{R})}<r, \mathcal{L}(w)>\delta / 4\right\}
$$

Note that $\mathcal{J}_{s}(w)<2 s$ implies $s^{2} / \mathcal{L}(w)<2 s$ and $\mathcal{L}(w)>s / 2>\delta / 4$.

Thus the map $s \rightarrow c(s) / s$ sends negligible sets to negligible sets. Since the function $t \rightarrow h(t)$ is non increasing on the interval $I$, its range $\mathcal{S}$ is Borel measurable (in fact $\overline{\mathcal{S}} \backslash \mathcal{S}$ is at most countable, like the set of discontinuity points of $h$ ). As the map $s \rightarrow c(s) / s$ is locally Lipschitz on $\mathcal{S}$ and $I$ has positive measure, we conclude that $\mathcal{S}$ has positive measure. This proves (a).

For $\mu \in \mathcal{S}$, we also get the estimate $\mu / \mathcal{L}(w)<1$ for all $w \in U \backslash\{0\}$ such that $\mathcal{J}_{\mu}(w)=c(\mu)$ :

$$
\begin{aligned}
0 & \stackrel{\mu \in \mathcal{S}}{\geq} \limsup _{s \rightarrow 1^{-}}(s-1)^{-1}\left\{\frac{c(s \mu)}{s \mu}-\frac{c(\mu)}{\mu}\right\} \\
& \geq \lim _{s \rightarrow 1^{-}}(s-1)^{-1}\left\{\frac{\mathcal{J}_{s \mu}(\sqrt{s} w)}{s \mu}-\frac{\mathcal{J}_{\mu}(w)}{\mu}\right\} \\
& =\left.\frac{d}{d s} \frac{\mathcal{J}_{s \mu}(\sqrt{s} w)}{s \mu}\right|_{s=1}=\left.\frac{d}{d s} \frac{\mathcal{K}(\sqrt{s} w)+s^{2} \mu^{2} \mathcal{L}^{-1}(\sqrt{s} w)}{s \mu}\right|_{s=1} \\
& =\frac{\mathcal{J}_{\mu}^{\prime}(w) w}{2 \mu}+\frac{2 \mu}{\mathcal{L}(w)}-\frac{\mathcal{J}_{\mu}(w)}{\mu}=\frac{2 \mu}{\mathcal{L}(w)}-\frac{\mathcal{J}_{\mu}(w)}{\mu}
\end{aligned}
$$


Hence

This proves $(\mathrm{d})$.

$$
\frac{\mu}{\mathcal{L}(w)} \leq \frac{c(\mu)}{2 \mu} \leq 1-\frac{\kappa \mu^{2}}{2}<1
$$

In the concentration-compactness, it is usual to prove the following property.

Property (SUB-H) at $\mu>0$ : strict subhomogeneity.

$$
c(t)<s c\left(s^{-1} t\right) \text { for all } s>1 \text { and } 0<t \leq \mu .
$$

It is unsettled in our examples if it holds for some small $\mu$. However, in the proof of the theorem above, a simple modification of it is shown to hold for many values of $\mu$. Strict subhomogeneity is equivalent to requiring that $t \rightarrow c(t) / t$ be strictly decreasing on $(0, \mu]$. As well known [12], [13], strict subhomogeneity implies strict subadditivity:

$$
c(\mu)=\mu_{1} \frac{c(\mu)}{\mu}+\mu_{2} \frac{c(\mu)}{\mu}<\mu_{1} \frac{c\left(\mu_{1}\right)}{\mu_{1}}+\mu_{2} \frac{c\left(\mu_{2}\right)}{\mu_{2}}=c\left(\mu_{1}\right)+c\left(\mu_{2}\right)
$$

for all $\mu_{1}, \mu_{2}>0$ such that $\mu_{1}+\mu_{2}=\mu$. In the first example, it is known to hold in the case $\beta \geq 1 / 3$ and $\mu$ small. However, in the case of interest in this paper (that is, $\beta \in(0,1 / 3))$, it is unsettled if it holds.

\section{Appendix: the second example}

The second example is dealt with in [5], but using a different minimization procedure and without studying minimizing sequences. Therefore, even if the computations are similar to those in [4]-[6], we feel that it is worth giving more details. The critical points of $\mathcal{J}_{\mu}$ satisfy

$$
\mathcal{K}^{\prime}(u)=\frac{\mu^{2}}{\mathcal{L}(u)^{2}} \mathcal{L}^{\prime}(u)
$$

the solutions of which correspond to solitary capillary-gravity water waves on the surface of an ocean of infinite depth. Their propagation speeds are proportional to $\mu / \mathcal{L}(u)$ (the constant of proportionality being independent of $\mu$ and the minimizer $u$ ). For related problems with finite depth, this implies some kind of weak stability for the set of minimizers as a whole [4], [6], [14], and its seems likely that some analogous stability result could also be deduced in the present case.

Let us first sketch the proof that the inequality in the subcriticallity hypothesis (SUB-C) holds:

$$
\exists \mu_{0}>0 \exists \kappa>0 \forall \mu \in\left(0, \mu_{0}\right] \quad c(\mu) \leq 2 \mu-\kappa \mu^{3} .
$$

For $\phi, \psi \in C_{0}^{\infty}(\mathbb{R})$ to be chosen later and $\alpha>0$ small, we define

$$
u(t)=\alpha \phi(\alpha t) \cos (t)+\alpha^{2} \psi^{2}(\alpha t) \cos 2 t
$$


and $\mu=\mathcal{L}(u)$. On page 513 in [5], we obtained the following estimates:

$$
\begin{gathered}
\sqrt{u^{\prime 2}+\left(1+\mathcal{H} u^{\prime}\right)^{2}}-\left(1+\mathcal{H} u^{\prime}\right)=\frac{1}{2} u^{\prime 2}-\frac{1}{2} u^{\prime 2} \mathcal{H} u^{\prime}-\frac{1}{8} u^{\prime 4}+\frac{1}{2} u^{\prime 2}\left(\mathcal{H} u^{\prime}\right)^{2}+\ldots, \\
\int_{\mathbb{R}} u^{\prime 2} d t=\frac{\alpha}{2} \int_{\mathbb{R}} \phi^{2} d t+\frac{\alpha^{3}}{2} \int_{\mathbb{R}} \phi^{\prime 2} d t+2 \alpha^{3} \int_{\mathbb{R}} \psi^{2} d t+O\left(\alpha^{4}\right), \\
\int_{\mathbb{R}} u^{2} d t=\frac{\alpha}{2} \int_{\mathbb{R}} \phi^{2} d t+\frac{\alpha^{3}}{2} \int_{\mathbb{R}} \psi^{2} d t+O\left(\alpha^{4}\right), \\
\int_{\mathbb{R}} u \mathcal{H} u^{\prime} d t=\frac{\alpha}{2} \int_{\mathbb{R}} \phi^{2} d t+\alpha^{3} \int_{\mathbb{R}} \psi^{2} d t+O\left(\alpha^{4}\right), \\
\int_{\mathbb{R}} u^{\prime 2} \mathcal{H} u^{\prime} d t=\frac{\alpha^{3}}{2} \int_{\mathbb{R}} \phi^{2} \psi d t+O\left(\alpha^{4}\right), \\
\int_{\mathbb{R}} u^{2} \mathcal{H} u^{\prime} d t=\alpha^{3} \int_{\mathbb{R}} \phi^{2} \psi d t+O\left(\alpha^{4}\right), \\
\int_{\mathbb{R}} u^{\prime 4} d t=\frac{3 \alpha^{3}}{8} \int_{\mathbb{R}} \phi^{4} d t+O\left(\alpha^{4}\right), \\
\int_{\mathbb{R}} u^{\prime 2}\left(\mathcal{H} u^{\prime}\right)^{2} d t=\frac{\alpha^{3}}{8} \int_{\mathbb{R}} \phi^{4} d t+O\left(\alpha^{4}\right) .
\end{gathered}
$$

We deduce

$$
\begin{aligned}
\mathcal{J}_{\mu}(u)-2 \mu= & \mathcal{K}(u)+\frac{\mu^{2}}{\mathcal{L}(u)}-2 \mu=\mathcal{K}(u)-\mathcal{L}(u) \\
= & \alpha\left\{\frac{1}{4}+\frac{1}{4}-\frac{1}{2}\right\} \int_{\mathbb{R}} \phi^{2} d t \frac{\alpha^{3}}{4} \int_{\mathbb{R}} \phi^{2} d t \\
& +\alpha^{3}\left\{1+\frac{1}{4}-1\right\} \int_{\mathbb{R}} \psi^{2} d t+\frac{\alpha^{3}}{4} \int_{\mathbb{R}} \phi^{\prime 2} d t \\
& +\left(-\frac{1}{4}+\frac{1}{2}\right) \alpha^{3} \int_{\mathbb{R}} \phi^{2} \psi d t+\frac{\alpha^{3}}{16} \int_{\mathbb{R}} \phi^{4} d t-\frac{3 \alpha^{3}}{64} \int_{\mathbb{R}} \phi^{4} d t+o\left(\alpha^{3}\right) .
\end{aligned}
$$

Setting $\psi=-A \phi^{2}$ for some constant $A$, we thus get

$\mathcal{J}_{\mu}(u)-2 \mu=\alpha^{3}\left\{\frac{1}{4} \int_{\mathbb{R}} \phi^{2} d t+\frac{1}{4} \int_{\mathbb{R}}{\phi^{\prime}}^{2} d t\right\}+\alpha^{3}\left(\frac{1}{4} A^{2}-\frac{1}{4} A+\frac{1}{64}\right) \int_{\mathbb{R}} \phi^{4} d t+o\left(\alpha^{3}\right)$.

We can now choose $A>0$ such that

$$
\frac{1}{4} A^{2}-\frac{1}{4} A+\frac{1}{64}<0
$$

and then $\phi$ such that

$$
\frac{1}{4} \int_{\mathbb{R}} \phi^{2} d t+\frac{1}{4} \int_{\mathbb{R}} \phi^{\prime 2} d t+\left(\frac{1}{4} A^{2}-\frac{1}{4} A+\frac{1}{64}\right) \int_{\mathbb{R}} \phi^{4} d t<0 .
$$

Moreover,

$$
\mu=\frac{\alpha}{2} \int_{\mathbb{R}} \phi^{2} d t+O\left(\alpha^{3}\right) .
$$


This clearly implies

$$
\exists \mu_{0}>0 \exists \kappa>0 \forall \mu \in\left(0, \mu_{0}\right] \quad c(\mu) \leq 2 \mu-\kappa \mu^{3} .
$$

Finally let us explain how to get a bounded minimizing sequence as in hypothesis (BMS). Remember equation (19) in [5]: there exists a constant $C>0$ such that

$$
\left|\mathcal{H} u^{\prime}(t)\right| \leq C\{1+\operatorname{dist}(t, \operatorname{supp}(u))\}^{-3 / 2}\|u\|_{W^{2,2}(\mathbb{R})}
$$

for all $u \in W^{2,2}(\mathbb{R})$ with compact support ( $C$ being independent of the size of the support). For the first example, a larger power than $3 / 2$ is available (see equation (15) in [4]) and this larger power is used in the method of [4] to prove (BMS).

To get a larger power, instead of $\mathcal{H} u^{\prime}$ we consider $N_{\varepsilon} u$ defined as follows. For $u \in W^{2,2}(\mathbb{R})$ and small $\varepsilon>0$,

$$
\widehat{N_{\varepsilon} u}(s):=\frac{s \cosh (s / \varepsilon)}{\sinh (s / \varepsilon)} \widehat{u}(s) .
$$

The operator $N_{\varepsilon}$ is related to the water-wave problem when the depth is finite and proportional to $1 / \varepsilon$.

We then get an analogous estimate as (15) in [4]. Namely, for all $n \geq 1$, there exists a constant $C_{\varepsilon, n}>0$ such that, for all $u \in W^{2,2}(\mathbb{R})$ with compact support $\operatorname{supp}(u)$,

$$
\left|N_{\varepsilon} u(t)\right| \leq C_{\varepsilon, n}\{1+\operatorname{dist}(t, \operatorname{supp}(u))\}^{-n+(1 / 2)}\left\{\int_{\operatorname{supp}(u)}\left(u-u^{\prime \prime}\right)^{2} d s\right\}^{1 / 2}
$$

$\left(C_{\varepsilon, n}\right.$ is independent of the size of the support). Moreover,

$$
\lim _{\varepsilon \rightarrow 0^{+}} \sup \left\{\left|\frac{s \cosh (s / \varepsilon)}{\sinh (s / \varepsilon)\left(1+s^{2}\right)^{1 / 2}}-\frac{|s|}{\left(1+s^{2}\right)^{1 / 2}}\right|: s \in \mathbb{R}^{*}\right\}=0
$$

and therefore

$$
\lim _{\varepsilon \rightarrow 0^{+}} \sup \left\{\left\|N_{\varepsilon} u-\mathcal{H} u^{\prime}\right\|_{W^{1,2}(\mathbb{R})}: u \in W^{2,2}(\mathbb{R}),\|u\|_{W^{2,2}(\mathbb{R})} \leq 1\right\}=0 .
$$

For fixed small $\mu>0$, we now apply the method of Theorem 2.1 in [6] and of Lemma 9 in [4] to the functionals $u \rightarrow \mathcal{L}_{\varepsilon}(u):=\Lambda_{\varepsilon} \int_{\mathbb{R}} u \cdot N_{\varepsilon} u d t, \mathcal{K}_{\varepsilon}$ obtained from $\mathcal{K}$ by replacing each occurrence of $\mathcal{H}^{\prime}$ by $N_{\varepsilon}$, and $\mathcal{J}_{\mu, \varepsilon}(u)=\mathcal{K}_{\varepsilon}(u)+\mu^{2} \mathcal{L}_{\varepsilon}(u)^{-1}$. The constant

$$
\Lambda_{\varepsilon}=\min \left\{\left(s^{2}+1\right) \frac{\sinh (s / \varepsilon)}{s \cosh (s / \varepsilon)}: s \geq 0\right\}>0
$$

is chosen such that

$$
\inf _{u \neq 0} \frac{\mathcal{K}_{\varepsilon}^{\prime \prime}(0)(u, u)}{2 \mathcal{L}_{\varepsilon}(u)}=1
$$


It satisfies $\lim _{\varepsilon \rightarrow 0^{+}} \Lambda_{\varepsilon}=2$. Clearly $\mathcal{J}_{\mu, \varepsilon}$ is defined on $U \backslash\{0\}$ too (if $\varepsilon>0$ is small enough) and

$$
\lim _{\varepsilon \rightarrow 0^{+}} \sup \left\{\left|\mathcal{J}_{\mu, \varepsilon}(u)-\mathcal{J}_{\mu}(u)\right|: u \in U, \mathcal{L}(u)>\mu / 4\right\}=0 .
$$

Hence, in the proof of Theorem 2.1 in [6], the test function $u$ above for $\mathcal{J}_{\mu}$ can also be used for $\mathcal{J}_{\mu, \varepsilon}$ if $\varepsilon$ is small enough. As in Lemma 9 in [4], for small fixed $\mu>0$ and all small enough $\varepsilon>0$, we get a minimizing sequence $\left\{u_{n, \varepsilon}\right\} \subset U \backslash\{0\}$ of $\mathcal{J}_{\mu, \varepsilon}$ such that

$$
\limsup _{\varepsilon \rightarrow 0^{+}} \limsup _{n \rightarrow \infty}\left\|u_{n, \varepsilon}\right\|_{W^{2,2}(\mathbb{R})}<r .
$$

It is then easy to get a minimizing sequence $\left\{u_{n}\right\} \subset U \backslash\{0\}$ of $\mathcal{J}_{\mu}$ such that

$$
\limsup _{n \rightarrow \infty}\left\|u_{n}\right\|_{W^{2,2}(\mathbb{R})}<r .
$$

Acknowledgements. This work started when J. F. Toland (University of Bath, UK) expressed his conviction that a set $\mathcal{S}$ of positive measure should be obtained.

\section{REFERENCES}

[1] K. I. BABEnKo, On a local existence theorem in the theory of surface waves of finite amplitude, Soviet Math. Dokl. 35 (1987), 647-650.

[2] - Some remarks on the theory of surface waves of finite amplitude, Soviet Math. Dokl. 35 (1987), 599-603.

[3] J. L. Bona And Yi A. Li, Decay and analyticity of solitary waves, J. Math. Pures Appl. 76 (1997), 377-430

[4] B. Buffoni, Existence and conditional energetic stability of capillary-gravity solitary water waves by minimisation, Arch. Rational Mech. Anal. 173 (2004), 25-68.

[5] _ Existence by minimisation of solitary water waves on an ocean of infinite depth, Ann. Inst. H. Poincaré Anal. Non Linéaire 21 (2004), 503-516.

[6] - Conditional energetic stability of capillary-gravity solitary water waves in the presence of weak surface tension, Topol. Methods Nonlinear Anal. 25 (2005), 41-68.

[7] B. Buffoni, É. SÉrÉ And J. F. Toland, Surface water waves as saddle points of the energy, Calc. Var. Partial Differential Equations 17 (2003), 199-220.

[8] - Minimisation methods for quasi-linear problems, with an application to periodic water waves, SIAM J. Math. Anal. 36 (2005), 1080-1094.

[9] T. Cazenave And P. L. Lions, Orbital stability of standing waves for some nonlinear Schrödinger equations, Commun. Math. Phys. 85 (1982), 549-561.

[10] A. I. Dyachenko, E. A. Kuznetsov, M. D. Spector and V. E. Zakharov, Analytic description of the free surface dynamics of an ideal fluid (canonical formalism and conformal mapping), Phys. Lett. A 221 (1996), 73-79.

[11] G. Iooss AND P. KirRmann, Capillary gravity waves on the free surface of an inviscid fluid of infinite depth, Existence of solitary waves, Arch. Rational Mech. Anal. 136 (1998), 1-19.

[12] P. L. Lions, The concentration-compactness principle in the calculus of variations-the locally compact case. 1, Ann. I. H. Poincaré Anal. Non Linéaire 1 (1984), 109-145. 
[13] - The concentration-compactness principle in the calculus of variations-the locally compact case. 2, Ann. I. H. Poincaré Anal. Non Linéaire 1 (1984), 223-283.

[14] A. Mielke, On the energetic stability of solitary water waves, Philos. Trans. Roy. Soc. London Ser. A 360 (2002), 2337-2358.

[15] S. Smale, Topology and mechanics I, Invent. Math. 10 (1970), 305-331.

[16] Topology and mechanics II, Invent. Math. 11 (1970), 45-64.

[17] V. Zakharov, Stability of periodic waves of finite amplitude on the surface of a deep fluid, J. Appl. Mech. Tech. Phys. 9 (1968), 190-194.

Manuscript received July 15, 2008

BORIS BUFFONI

Section de Mathématiques (IACS)

École Polytechnique Fédérale

Station 8

CH-1015 Lausanne, SWITZERLAND

E-mail address: Boris.Buffoni@epfl.ch 